\title{
ERYTHROCYTES AS A TARGET OF SARS COV-2 IN PATHOGENESIS OF COVID-19
}

Received 01 July 2020;

Received in revised form 05 August 2020; Accepted 07 August 2020

\section{Ivan Reva ${ }^{1 凶}\left(\mathbb{D}\right.$, Tatsuo Yamamoto ${ }^{1}$ (D), Mariya Rasskazova ${ }^{2}$, Tatyana Lemeshko3, Victor Usov² (D, Yuriy Krasnikov'², Anna Fisenko33, Evgeniy Kotsyurbiy3, Vladislav Tudakov', Ekaterina Tsegolnik ${ }^{3}$, Olesya Oleksenko ${ }^{3}$, Anatoly Korobkin², Ellada Slabenko², Anastasiya Shindina' ${ }^{2}$ Kseniya Gordzievskaya ${ }^{2}$, Anna Furgal ${ }^{2}$, Galina Reva ${ }^{1,2}$ (D),}

\author{
${ }^{1}$ International Medical Research Center, Niigata, Japan, \\ ${ }^{2}$ Far Eastern federal university, Vladivostok; \\ ${ }^{3}$ Pacific State Medical University, Vladivostok, Russia
}

avers2@yandex.ru

\begin{abstract}
The paper considers the possible mechanism of the pathogenesis of COVID-19 caused by SARSCOV-2, associated with damage to red blood cells, which the authors attribute to the main key target that triggers a cascade of reactions leading to multiple organ failure. The paper presents morphological evidence for the presence of pathological forms of erythrocytes characteristic of various anemias in the blood vessels and parenchyma of damaged lungs of patients with COVID-19. The death of red blood cells leads to cell ischemia and anemia. The defeat of brain neurons, blood vessels and hematotissue barriers in organ systems is a consequence of ischemia due to the impossibility of transferring hemoglobin by damaged erythrocytes and ends at the terminal stages of the development of the disease with their dysfunction. Adaptive erythropoiesis with an increase in erythropoietin secretion is especially dangerous for patients suffering from hypertension, and then it is impossible, since all organs involved in the synthesis of erythropoietin are damaged. In this case, the synthesis of hemoglobin is also disrupted due to a deficiency of iron and cyancobolamin, whereas toxic iron and hemosiderin are deposited in the tissues.
\end{abstract}

KEY W OR DS - COVID-19, SARS-COV-2, erythrocytes, hemoglobin, erythropoiesis, viruses, pathogenesis of COVID-19.

\section{RELEVANCE}

In the world, the number of cases of COVID-19 has reached 16.4 million. The number of coronavirus victims worldwide exceeds 600 thousand, including children $[1,5,16]$. Madabhavi I., Sarkar M., Kadakol N. (2020) identified the pandemic as a catastrophe of global significance [6], which indicates the high urgency of the problem under study $[9,12,21]$. It is generally accepted that viruses multiply in the cell, specializing in certain cell types: HIV infects Thlymphocytes, the influenza virus infects the epithelium of the larynx and lungs, coronavirus destroys the epithelium of the upper respiratory tract, lungs and gastrointestinal tract, herpes affects the nerves and causes neuroinfections, hepatitis viruses infect liver cells, etc. $[17,20]$. With COVID-19 caused by SARS-COV-2, multiple organ disorders occur in various structures, including nerve structures $[3,14]$. Burness A. T. and Pardoe I. U. (1981) established the possibility of attachment of the influenza virus to human erythrocytes, followed by hemagglutination of the cells [4]. Allaway G.P., Burness A.T. (1986) showed that the main type of glycophorins, glycophorin A, is a receptor for the attachment of influenza and encephalomyocarditis (EMS) viruses to human erythrocytes $[2,4]$. The attachment of the EMS virus to glycophorin A includes a region containing amino acids 35 to about 70 (numbered from the end of NH2), four of which (amino acids 37, 44, 47 and 50) are glycosylated. In addition, there is evidence that segments containing amino acids 35 to 39 with an oligosaccharide side chain on threonine-37 are particularly important for the attachment of EMS virus. However, in the available scientific literature against the background of the available pathomorphological data on the destruction of lung tissue, death of alveolocytes, fibrotic changes in the lungs, there is no assessment of erythrocytes located both in the lumen of blood vessels and released into the parenchyma of affected organs $[5,19,24]$. Given the multiple organ failure that develops in COVID-19 [7, 18], the absence of a key morphological target for SARS-COV-2 [8], as well as controversial data on the nature of the virus itself, we directed our research to study changes in the red blood cell pool against the background of COVID-19. According to Rodríguez-Nóvoa S., Morello J., González M., et al (2008), the use of antiviral drugs causes hemolysis and increases hyperbilirubinemia [13]. According to them, treatment of HIV/hepatitis-C infected patients led to an increase in hyperbilirubinemia from $9 \%$ to $45 \%$ after starting treatment for hepatitis C. Anemia has a multifactorial nature $[10,15]$, which explains the 
cases of unsuccessful attempts to empirically use erythropoietin in the treatment of patients with hepatitis $C$ and HIV-infected [11]. All this dictates an expansion of the range of measures aimed at studying the etiology and pathogenesis of COVID-19 for the development of pathogenetically justified prevention and treatment that is absent at the present stage $[22,23]$.

\section{Purpose of the study:}

To study the role of red blood cells in the pathogenesis of COVID-19.

\section{MATERIAL AND METHODS}

The paper analyzes and discusses the results of our own studies of lung biopsies of 11 patients who died from COVID-19 caused by SARS-CoV-2, with PCR confirmation, obtained in accordance with the order of the Ministry of Healthcare of the Russian Federation dated 04.29.94 N 82 On the procedure for conducting pathological autopsies, which regulates the procedure for autopsies in medical institutions. The control group consisted of 14 patients who died as a result of injuries incompatible with life, presumably without somatic pathology at the age of 24 to 76 years. The biopsy specimens were fixed immediately after collection in accordance with the preparation protocol for histological examination. The exclusion of possible artifacts is based on data obtained during a special study on dogs, indicating that when corpses are stored at a temperature of $4-7^{\circ} \mathrm{C}$ for $4-6$ hours, microscopically visible changes in the morphology of various organ systems are not observed, except for a slight decrease in the intensity of specific reactions on enzymatic activity. We used classical histological research methods with hematoxylin-eosin staining to obtain a general morphological picture.

The material was analyzed using an OlympusBx82 microscope and a CDx82 digital camera with proprietary software. The morphological method of the study consisted in the preparation of sections from paraffin blocks of lung biopsies from SARS-CoV-2 victims, followed by staining with hematoxylin and eosin in accordance with the classical protocol. Analysis of preparations and production of illustrations were carried out using an Olympus Bx52 microscope and a DP25 digital camera.

\section{RESULTS OF OUR OWN RESEARCH}

We have found that erythrocytes are one of the targets damaged by SARS-COV-2. Anisocytosis and poikilocytosis of erythrocytes are noted, macrophages with hemosiderin, hypochromic and hyperchromic erythrocytes are identified. Erythrocytes in the form of a sickle are identified (in Fig. 1 it is indicated by an arrow), as well as spherocytes, macro and microcytes. The appearance of sickle erythrocytes may be associated with disturbances in the B-chains of globin and the replacement of glutamine with valine. The solubility of such globin decreases 25 times, depending on the partial pressure of oxygen in the blood and the concentration of $\mathrm{HbS}$ in the erythrocyte. The distribution of hemoglobin in the cytoplasm of erythrocytes is uneven, bright oxyphilia is observed both in the center of erythrocytes and in the periphery. Thickening of the erythrocyte membrane, chromophobic courtyard around the red blood cells. Kebot's rings are identified, staining red. Kebot's rings are characteristic and are found mainly in megaloblastic anemia and metal intoxication $(\mathrm{Pb})$. Under COVI-19 conditions, this toxic effect is exerted by the hemoglobin of dying erythrocytes, transferrin and iron, as well as hemosiderin. We have established that Howell-Jolly bodies are identified on lung preparations, which are small round violet-red inclusions 1-2 microns in size, less often found in 2-3 in one erythrocyte. It is known that Howell-Jolly bodies are the remainder of the erythrocyte nucleus after removal of its RES. Revealed more often with intense hemolysis and with megaloblastic anemia (Fig. 1).

Some of the erythrocytes have a swollen membrane at one of the poles with the formation of a vacuole (Figure 1, indicated by blue arrows). In addition, all degenerative forms of erythrocytes with a characteristic location of hemoglobin are observed: hypochromic with a hole, ring, and also in the form of shadows, indicating iron deficiency and B12 anemia; hyperchromic with an arrangement of hemoglobin in the form of a ring with and without enlightenment (Fig. 2).

This explains the severity of clinical manifestations when the gastrointestinal tract is involved in the pathological process, since the participation of cyancobolamine in the normalization of erythropoiesis is inhibited. The presence of spherocytes in the lung parenchyma and in the lumen of blood vessels indicates hyperhydration of the erythrocyte, increased permeability of the erythrocyte membrane for $\mathrm{Na}^{+}$ and $\mathrm{Ca}^{2+}$ ions, followed by the acquisition of spherical erythrocytes with the inability to plasticity and deformation in the microvasculature, with a decrease in life expectancy. It is known that with such changes, the developing membranopathy is mainly due to a significant change in the content of the spectrin protein, a violation of its binding to other proteins of the erythrocyte membrane. Since glycolysis is the leading pathway for ATP resynthesis in erythrocytes, a lack of ATP energy causes a violation of the transmembrane transfer of ions, a decrease in the activity of enzymes of the pentose phosphate cycle. Their imbalance develops, leading to overhydration and swelling of 

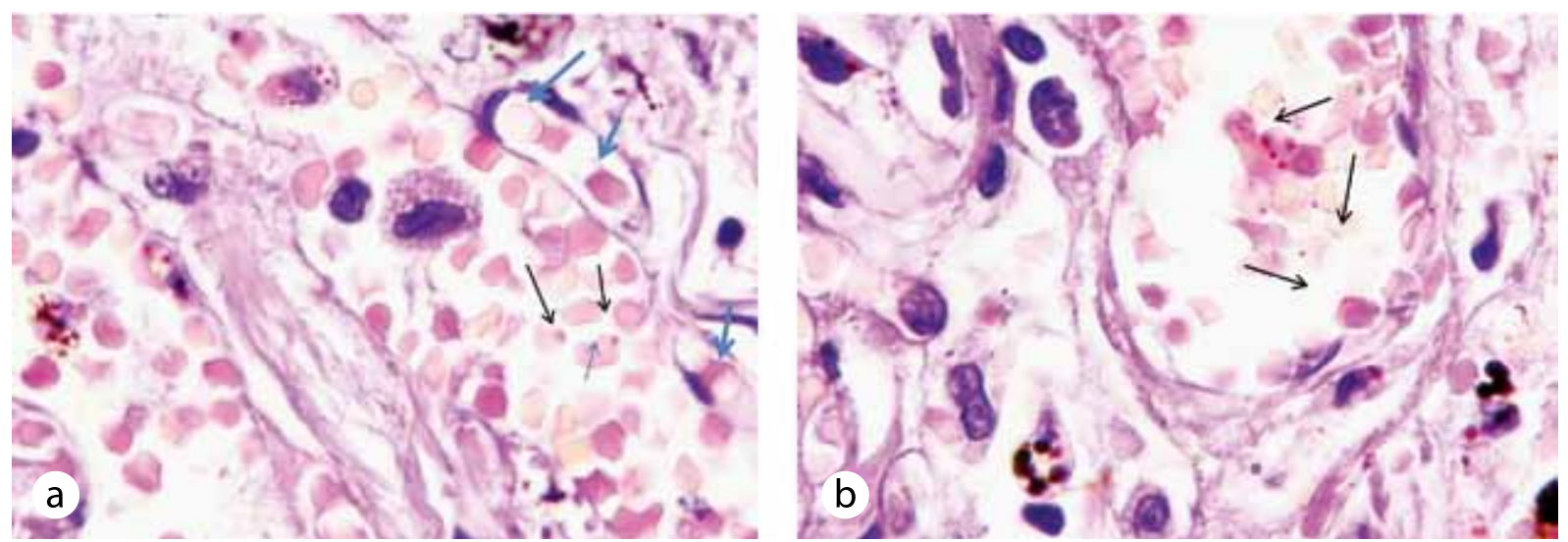

Fig. 1. Lung parenchyma of patients a) 53 years old; b) 74 years old with COVID-19. Staining with hematoxylin and eosin. Microphoto, $\times 400$. Howell-Jolly bodies are identified (indicated by black arrows)
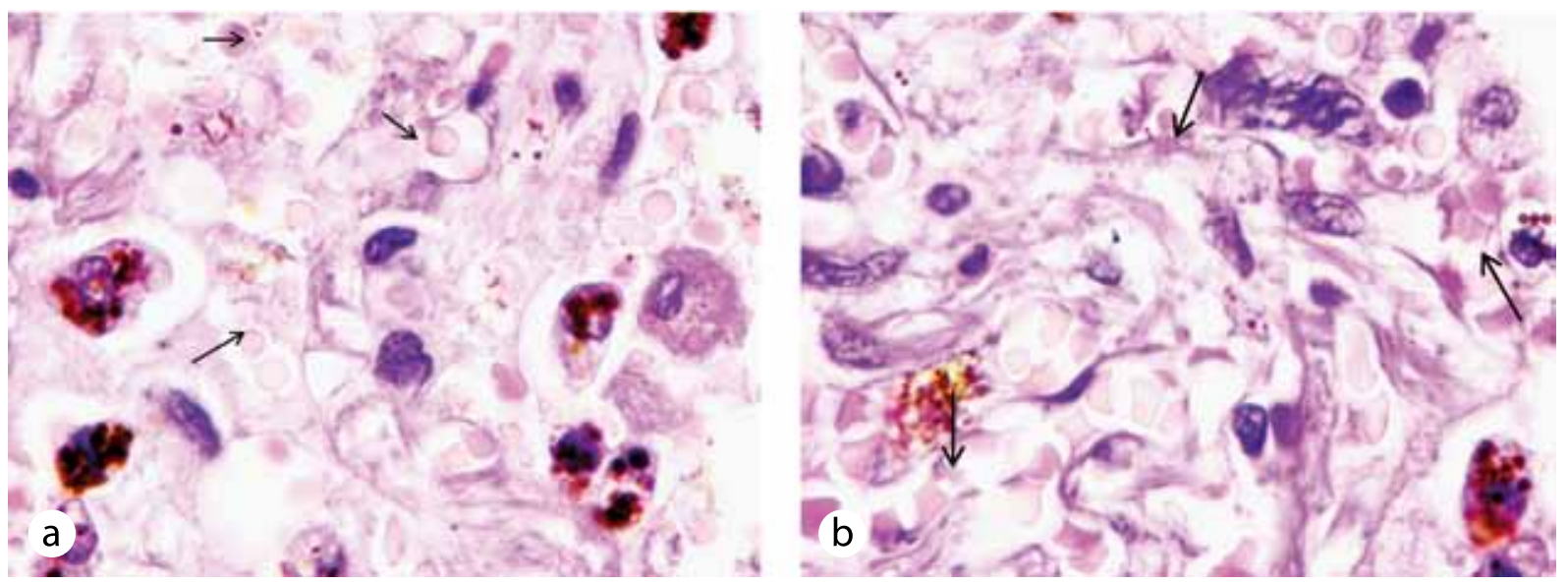

Fig. 2. Lung parenchyma of patients a) 56 years old; b) 64 years old with COVID-19. Staining with hematoxylin and eosin. Microphoto, $\times 400$. Spherocytes, microcytes, hypo- and hyperchromic erythrocytes are identified

erythrocytes. In addition, we noted the distribution of hemoglobin at opposite poles of cells or at one of the poles, typical for erythrocytes located both in the lumen of blood vessels and in the parenchyma of the lung. Also, cell nuclei are identified, the morphology of which is characteristic of those damaged by viruses, has a ring-shaped shape, or inclusions (Fig. 3).

Damage to erythrocytes does not have the character of artifacts resulting from non-technological implementation of methods in violation of the recommended protocol, as evidenced by the presence of normocytes, as well as a clear morphology of macrophages and other cells containing hemosiderin, a product of erythrocyte destruction (Fig. 4).

In addition, hemosiderin is contained not only in the cytolasm of macrophages, but also in the lumen of blood vessels (Fig. 5, 6).
The presence of macrophages with hemosiderin in the cytoplasm, as well as free hemosiderin in the lumen of blood vessels, indicate that the death of erythrocytes begins not in the lung parenchyma, but at the stage of circulation and oxygen delivery to the tissues. The consequence of this is the generalization of the pathological process, leading to multiple organ failure. Thus, for the causative agent of COVID-19 SARS COV-2 in starting the cascade of pathogenesis, one of the main targets is the erythrocyte, in which membranopathy, fermentopathy and hemoglobinopathy are clearly manifested. Adaptive erythrocytosis, an increase in the number of reticulocytes and arterial hypertension are accompanied by a decrease in blood plasma volume when the body loses fluid as a result of diarrhea, vomiting, and plasmorrhage. In our observations, 3 out of 11 patients developed anemia with a 

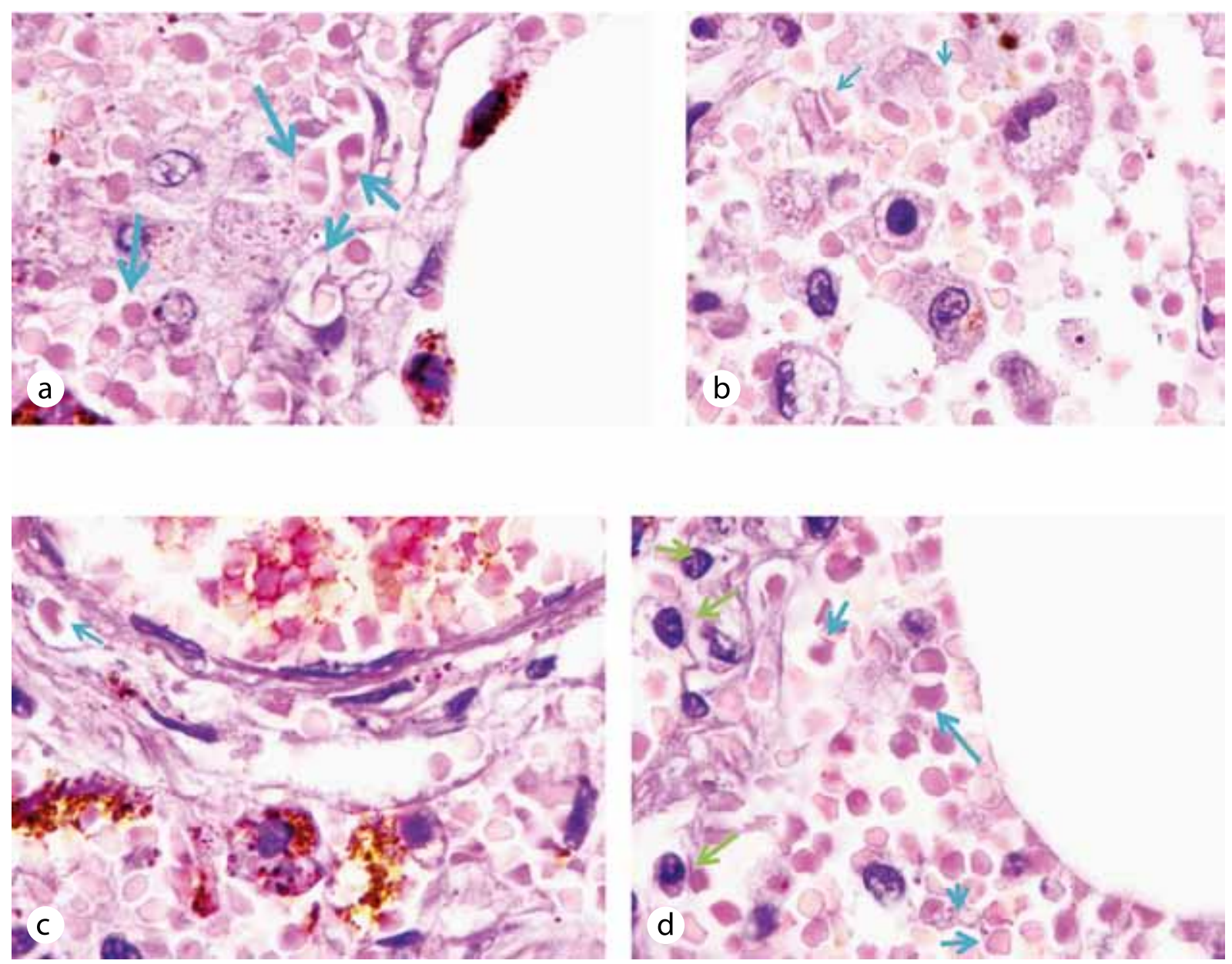

Fig. 3. Lung parenchyma of patients a) 39 years old; b) 56 years old; c) 64 years old; d) 71 years old with (OVID-19. Staining with hematoxylin and eosin. Microphoto, $\times 400$. Spherocytes, microcytes, hypo-and hyperchromic erythrocytes are identified. The uneven distribution of hemoglobin at the poles of eryrocytes is indicated by blue arrows, and the nuclei of cells affected by viral RNA are indicated by green arrows
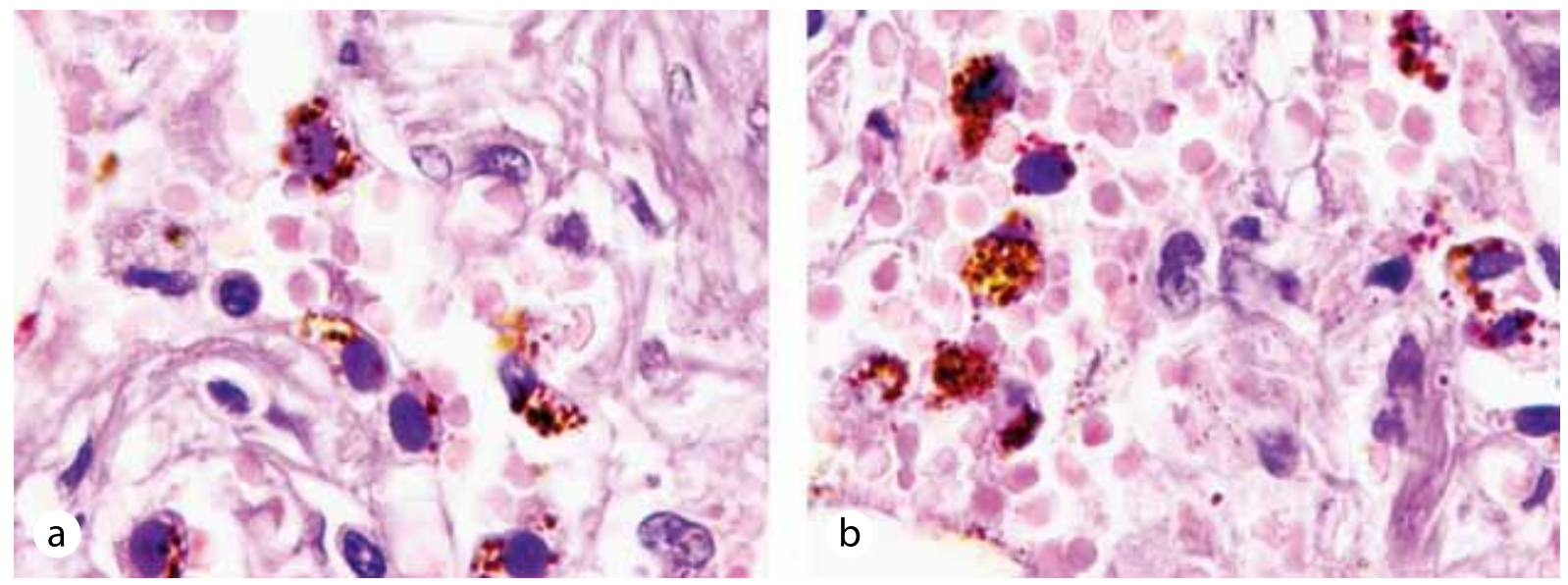

Fig. 4. Lung parenchyma of patients a) 51 years old; b) 56 years old with COVID-19. Staining with hematoxylin and eosin. Microphoto 

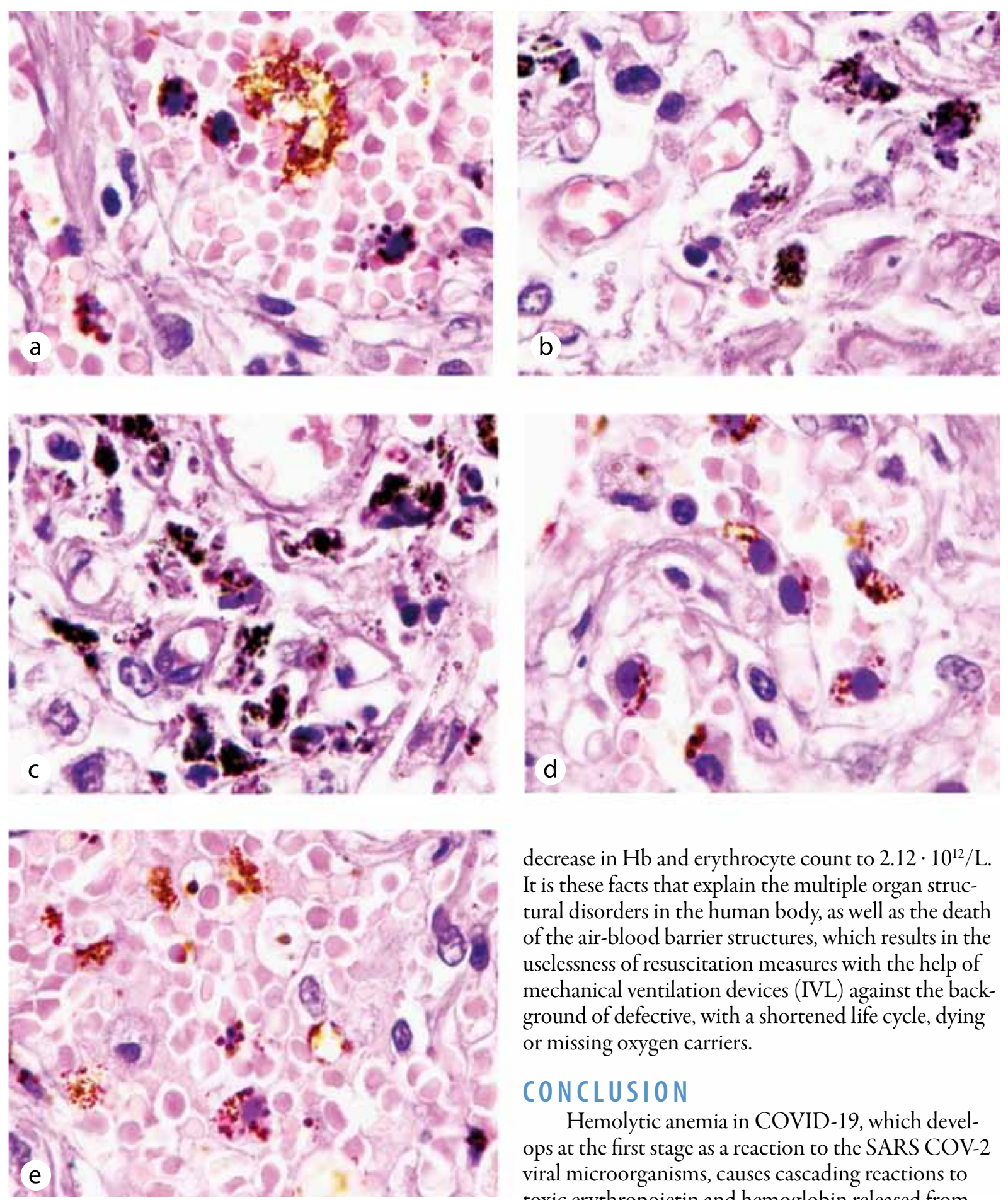

decrease in $\mathrm{Hb}$ and erythrocyte count to $2.12 \cdot 10^{12} / \mathrm{L}$. It is these facts that explain the multiple organ structural disorders in the human body, as well as the death of the air-blood barrier structures, which results in the uselessness of resuscitation measures with the help of mechanical ventilation devices (IVL) against the background of defective, with a shortened life cycle, dying or missing oxygen carriers.

\section{CONCLUSION}

Hemolytic anemia in COVID-19, which develops at the first stage as a reaction to the SARS COV-2 viral microorganisms, causes cascading reactions to toxic erythropoietin and hemoglobin released from erythrocytes in the bloodstream, and then to hemosiderin released due to the death of erythrocytes in the tissue. The process ends with a decrease in the synthesis of erythropoietin in the decaying liver and kidneys, in the absence of the necessary vitamin B12 due to the pathology of its secretion in the gastrointestinal tract. The characteristic signs of damage to red blood cells in conditions of infection with COVID-19 indicate 

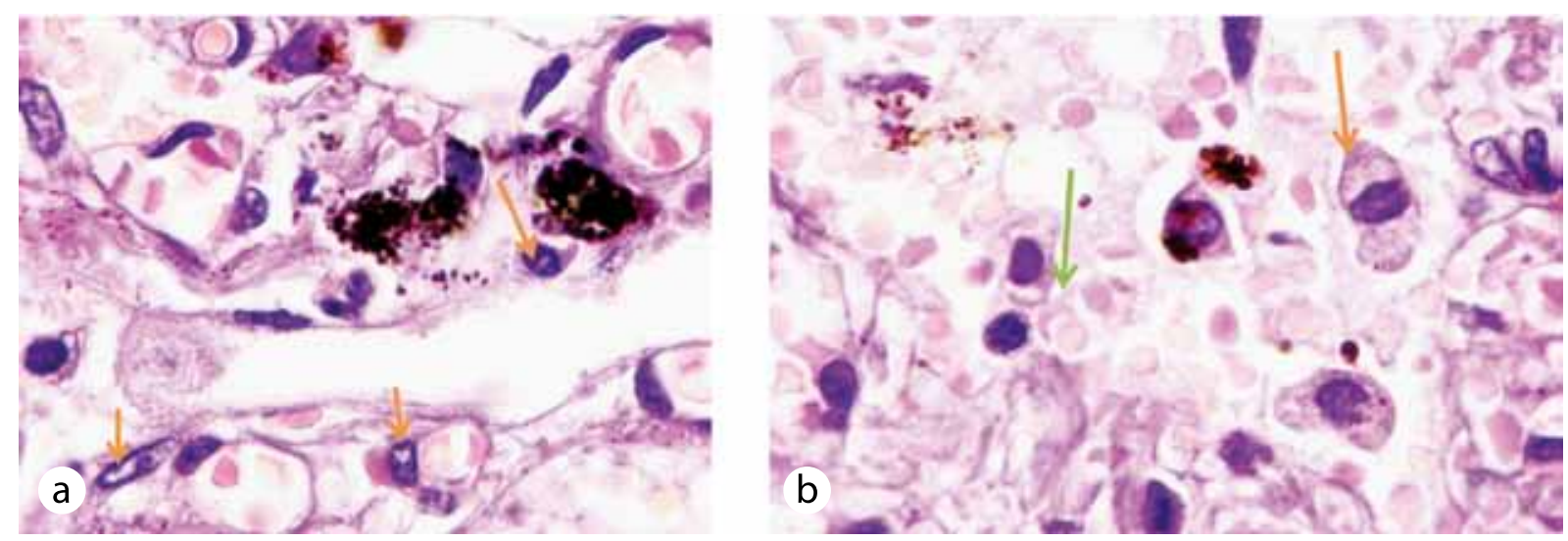

Fig. 6. Lung parenchyma of patients a) 64 years old; b) 71 years old with COVID-19. Staining with hematoxylin and eosin. Microphoto, $\times 400$. (Green arrow indicates sickle-shaped erythrocyte. Orange arrows indicate viral inclusions in cell nuclei)

disruption of erythropoiesis, with developing iron deficiency and B12 anemia. The synthesis of hemoglobin lags behind the differentiation of erythrocytes, which in the initial period of the disease induces an increase in the secretion of erythropoietin, which has a hypertensive effect; increases blood viscosity and leads to impaired blood microcirculation, which is why patients with essential hypertension are the main risk group. This underlies the inability to use erythropoietin-based drugs to treat patients with anemia in the context of COVID-19 infection. Pathological changes in erythrocytes infected with viruses are caused by specific and non-specific processes. Reactions caused by the configuration of the permeability of the plasma membrane of erythrocytes, margination of chromatin in the form of Howell-Jolly bodies, and vacuolization of the cytoplasm are nonspecific. SARS COV-2, like SV40, can be attributed to vacuolizing viruses, but only erythrocytes, since the virus has a particularly peculiar and pronounced character of inducing vacuolization. Specific changes are oxyphilic viral inclusions in cell nuclei, leading to cell destruction through a rapid and deep effect on the synthesis of cellular proteins.

\section{CONCLUSIONS}

1. The key target for SARS COV-2 is erythrocytes.

2. Multiorgan failure is the result and consequence of SARS COV-2 virus damage to erythrocytes, which develops against this background of ischemia and anemia with cell death of various organ systems.

3. The use of erythrocyte mass for the treatment of COVID-19 is pathogenetically substantiated.

The study was carried out within the framework of the state assignment $17.5740 .2017 / 6.7$, the international
Russian-Japanese grant of the FEFU (agreement No. 1309-0602-m_a dated 06.11.2013) and the execution of the grant from the Russian Science Foundation 19-14-00260 (2019).

\section{REFERENCES}

1. Ahn D.G., Shin H.J., Kim M.H., Lee S., Kim H.S., Mroung J., Kim B.T., Kim S.J. Current Status of Epidemiology, Diagnosis, Therapeutics, and Vaccines for Novel Coronavirus Disease 2019 (COVID-19).//J Microbiol Biotechnol. 2020 Mar 28;30(3):313-324. doi: $10.4014 / \mathrm{jmb} .2003 .03011$.

2. Allaway G.P., BuRness A.T.J Site of attachment of encephalomyocarditis virus on human erythrocytes.//Virol. 1986 Sep;59(3):768-70. doi: 10.1128/ JVI.59.3.768-770.1986.

3. Buja L.M., Wolf D.A., Zhao B., AkKanti B., McDonald M., Lelenwa L., Reilly N., OTtaViani G., Elghetany M.T., Trujillo D.O., AISENBERG G.M., MADJID M., KaR B. The emerging spectrum of cardiopulmonary pathology of the coronavirus disease 2019 (COVID-19): Report of 3 autopsies from Houston, Texas, and review of autopsy findings from other United States cities.//Cardiovasc Pathol. 2020 Sep-Oct; 48:107233. doi: 10.1016/j. carpath.2020.107233

4. Burness A. T., Pardoo I. U. Effect of enzymes on the attachment of influenza and encephalomyocarditis viruses to erythrocytes.// J Gen Virol. 1981 Aug;55(Pt 2): 275-88. doi: 10.1099/0022-1317-55-2275. DOI: $10.1099 / 0022-1317-55-2-275$

5. Jiang F., Deng L., Zhang L., Cai Y., Cheung C.W., XIA Z. Review of the Clinical Characteristics of Coronavirus Disease 2019 (COVID-19).Version 2.// J. Gen Intern Med. 2020 May;35(5):1545-1549. doi: 10.1007/s11606-020-05762-w.

6. Јоов B, Wiwanitkit V. Pulmonary Pathology of Early Phase 2019 Novel Coronavirus Pneumonia.//J. 
Thorac Oncol. 2020 May; 15(5):e67. doi: 10.1016/j. jtho.2020.03.013.PMID: 32340677

7. Kannan S., Shaik Syed Ali P., Sheeza A., Hemalatha K. COVID-19 (Novel Coronavirus 2019) - recent trends. //Eur Rev Med Pharmacol Sci. 2020 Feb;24(4):2006-2011. doi: 10.26355/eurrev_202002_20378.

8. LI Y.C., BaI W.Z., HaShikawa T. J. The neuroinvasive potential of SARS-CoV2 may play a role in the respiratory failure of COVID-19 patients.//Med Virol. 2020 Jun;92(6):552-555. doi: 10.1002/jmv.25728.

9. Madabhavi I., Sarkar M., Kadakol N. COVID-19: a review.//Monaldi Arch Chest Dis. 2020 May 14;90(2). doi: 10.4081/monaldi.2020.1298.

10. Ochoa D., Redondo T., Moreno-Rueda G. Mizutama: A Quick, Easy, and Accurate Method for Counting Erythrocytes.//Physiol Biochem Zool. 2019 Mar/Apr;92(2):206-210. doi: 10.1086/702666.

11. Reva I.V., Reva G.V., Yamamoto T.T., OleKSENKo O.M., RassKazova M.E., Reva G.V., OleKSENKo O.M., RasSKazova M.E., REVA I V.V., Reva G.V., Yamamoto T.T., Gulkov A.N., OleKSENKo O.M., Rasskazova M.E., Danilenko M.V., Vershinina S.S., Shmelev M.E., Tyasto V.A. The role of ischemia in the pathogenesis of hepatitis c complicated by glomerulonephritis and carcinogenesis // Modern problems of science and education. - 2015. - No. 5; URL: http://science-education.ru/ru/article/view?id=22049; DOI: 10.17513/ spno.128-22049

12. Rico-Mesa J.S., White A., ANderson A.S. Outcomes in Patients with COVID-19 Infection Taking ACEI/ARB.//Curr Cardiol Rep. $2020 \mathrm{Apr}$ 14;22(5):31. doi: 10.1007/s11886-020-01291-4.

13. Rodríguez-Nóvoa, S., Morello, J., GonZález, M., Vispo, E., Barreiro, P., González-Pardo, G., JimÉnEZ-NÁCHER, I., GONZALEZ-LahoZ, J., SorIano, V. Increase in serum bilirubin in HIV/ hepatitis-C virus-coinfected patients on atazanavir therapy following initiation of pegylated-interferon and ribavirin.//AIDS. 2008 Nov 30;22(18):2535-7. doi: 10.1097/QAD.0b013e3283177f38.

14. Shanmugaraj B., Siriwattananon K., Wangkanont K., Phoolcharoen W. Perspectives on monoclonal antibody therapy as potential therapeutic intervention for Coronavirus disease-19 (COVID-19).//Asian Pac J Allergy Immunol. 2020 Mar;38(1):10-18. doi: 10.12932/AP-200220-0773.

15. Shaz B.H. Red blood cells: beyond the transfusion.// Blood. 2019 Jun 20;133(25):2627-2628. doi: 10.1182/ blood-2019-04-901140.

16. SHe J, Liu L, LiU W. COVID-19 epidemic: Disease characteristics in children.//J Med Virol. 2020 Jul;92(7):747-754. doi: 10.1002/jmv.25807.

17. Sun P., Lu X., Xu C., Sun W., Pan B. Understanding of COVID-19 based on current evidence.//J Med Virol. 2020 Jun;92(6):548-551. doi: 10.1002/ jmv.25722.
18. Tian S., Hu W., Niu L., Liu H., Xu H., Xiao S.Y. Pulmonary Pathology of Early-Phase 2019 Novel Coronavirus (COVID-19) Pneumonia in Two Patients With Lung Cancer.//J. Thorac Oncol. 2020 May;15(5):700-704. doi: 10.1016/j.jtho.2020.02.010.

19. Tian S., Xiao S.Y. Pathology of 2019 Novel Coronavirus Pneumonia: A Dynamic Disease Process.//J. Thorac Oncol. 2020 May;15(5):e67-e68. doi: 10.1016/j.jtho.2020.03.015

20. Wajcman H., Kiger L.C. R. Hemoglobin, from microorganisms to man: a single structural motif, multiple functions// Biol. 2002 Dec;325(12):1159-74. doi: 10.1016/s1631-0691(02)01537-8.

21. Wang Y., Wang Y., Chen Y., Qin Q. Unique epidemiological and clinical features of the emerging 2019 novel coronavirus pneumonia (COVID-19) implicate special control measures./J Med Virol. 2020 Jun;92(6):568-576. doi: 10.1002/jmv.25748.

22. Wu Y., Xu X., Chen Z., Duan J., Hashimoto K., Yang L., Liu C., Yang C. Nervous system involvement after infection with COVID-19 and other coronaviruses.//Brain Behav Immun. 2020 Jul;87:18-22. doi: 10.1016/j.bbi.2020.03.031.

23. YANG L., Tian D., LiU W. Strategies for vaccine development of COVID-19.//Sheng Wu Gong Cheng Xue Bao. 2020 Apr 25;36(4):593-604. doi: 10.13345/j. cjb.200094.

24. Zhang L, Jiang Y, Wei M, Cheng BH, Zhou XC, Li J, Tian JH, Dong L, Hu RH. Analysis of the pregnancy outcomes in pregnant women with COVID-19 in Hubei Province.//Zhonghua Fu Chan Ke Za Zhi. 2020 Mar 25;55(3):166-171. doi: 10.3760/ cma.j.cn112141-20200218-00111 